\title{
SALT Spectroscopy of Dark Energy Survey Supernovae and their Classification
}

\author{
E. K. Kasai ${ }^{* a b c}$, B. A. Bassett ${ }^{a b c}$, S. M. Crawford ${ }^{b}$, M. Smith ${ }^{d}$ \\ ${ }^{a}$ African Institute for Mathematical Sciences, 6-8 Melrose Road, Muizenberg, 7945, South Africa \\ ${ }^{b}$ South African Astronomical Observatory, P.O.Box 9, Observatory 7935, South Africa \\ ${ }^{c}$ Department of Mathematics and Applied Mathematics, University of Cape Town, Rondebosch \\ 7700, South Africa \\ ${ }^{d}$ School of Physics and Astronomy, University of Southampton, Southampton SO17 1BJ, UK \\ E-mail: kasaiedsaao.ac.za, brucedsaao.ac.za, crawfordesaao.ac.za, \\ mat.smithesoton.ac.uk
}

\begin{abstract}
We present preliminary results of our SALT spectroscopic follow-up program of supernova (SN) candidates discovered by the international Dark Energy Survey. The results are equivalent width (EW) measurements of two type Ia supernova (SN Ia) spectral features: Ca II H\&K and Si II $4000 \AA$, in our sample size of 10 SN(e) Ia, 9 of which were observed in the redshift range $0.1<z<$ 0.3. Our measured EW values are found to be consistent with similar measurements performed on low-redshift $(z<0.1)$ SN Ia samples, suggesting no evolution in the EW of Ca II H\&K and Si II $4000 \AA$ SN Ia spectral features with $z$. Additionally, we provide a brief discussion and some results of a technique we have been developing to classify low signal to noise and host galaxy contaminated SALT spectra. We start the introduction with a discussion of the use of SNe Ia in the context of studying the accelerated expansion of the Universe and the exact behaviour of "dark energy" hypothesised to cause the accelerated expansion. We provide some examples of how EW measurements of SN Ia spectral features can play an important role in helping us gain a better understanding of systematic uncertainties that currently undermine the accuracy with which we estimate cosmological parameters from SN Ia data.
\end{abstract}

SALT Science Conference 2015 -SSC2015-

1-5 June, 2015

Stellenbosch Institute of Advanced Study, South Africa

${ }^{*}$ Speaker. 


\section{Introduction}

Observations of low and high redshift SNe Ia demonstrated back in 1998 that the Universe's rate of expansion was accelerating [1,2]. This discovery implied that either Einstein's theory of gravity is incomplete on large scales or there exists a dominant force-which we call "dark energy" - in the Universe to cause the observed accelerated expansion. Both hypotheses are to this day not well-understood and remain very active areas of research in Cosmology. The current best estimate for the value of dark energy from observations of SNe Ia is $\Omega_{\Lambda}=0.705 \pm 0.034$ (stat+sys) for a flat Universe [3].

The use of SNe Ia in estimating cosmological parameters rests on the phenomenological relationship between peak luminosity and light-curve width called the 'Phillips relation' (see [4] for details). Use of this relation allows $\mathrm{SNe}$ Ia to be treated as 'standadizable' candles with a remarkably low level of scatter in the inferred peak luminosity. Despite their standardizable candle reputation, subtle differences among SN Ia light curves and spectra are observed (see [5] for a review). In estimating the value of dark energy and other cosmological parameters, SN Ia light curves are standardized by matching each light-curve to a phenomenological SN Ia light-curve template determined from a training set of $\mathrm{SNe}$ Ia with known relative distances. The process involves the use of what are called light-curve fitters [6,7], which determine the best stretch $x_{1}$ (related to the width of the SN light-curve) and colour $c$ (colour of a supernova) parameters used in minimising the difference between a given light-curve and the template.

The accuracy of SN Ia light-curve standardization is however affected by several factors including dust extinction in the SNe's host galaxies. While the Cardelli extinction law [8] gives good estimates of extinction due to dust in the Milky Way (MW) and has a total-to-selective extinction average value of $R_{V}=3.1$, little is understood about this value in the host galaxies of observed SNe, with measurements by some authors placing the value between 1.1 and 2.8 inclusive $[9,10,11]$. The uncertainty caused by dust extinction in the derived $x_{1}$ and $c$ light-curve parameters prompted the community to look into the use of spectral parameters in standardizing SN Ia light curves. Using ratios of fluxes $\mathscr{R}$ measured at selected wavelengths of SN Ia spectra, [12] demonstrated that substituting $x_{1}$ and $c$ with $\mathscr{R}$ in standardizing light curves yielded less scatter in the Hubble diagram residuals. They also found that using all three parameters $\left(x_{1}, c\right.$ and $\left.\mathscr{R}\right)$ in the standardization decreased the scatter further. Their findings were corroborated by [13]. Using equivalent width (EW) measurements of the SN Ia Ca II H\&K and Si II 4000A spectral features, [11] derived an $R_{V}$ value of $2.8 \pm 0.3$, consistent with the value derived by [8] for the MW.

The use of spectral features in standardizing SN Ia light curves has however only been performed prolifically on low-redshift samples leaving open the question of whether or not the same results are attainable at intermediate and high redshifts. In this contribution, we present our preliminary results of EW measurements of the Ca II H\&K and Si II $4000 \AA$ spectral features of 10 SALT spectra of SN Ia candidates discovered by the Dark Energy Survey (DES, which aims to measure the expansion history of the Universe with unprecedented accuracy to place tighter constraints on the value of dark energy). The outline of our contribution is as follows: In Section 2, we briefly describe the DES observational set-up as well as our SALT spectroscopic follow-up campaign and the data reduction details. We discuss briefly in Section 3 the Bayesian SN classification method we developed and in Section 4, we describe the process of measuring EWs and present our prelimi- 
nary results. We discuss the results and how they compare to the literature in Section 5 and provide a conclusion and our plans for future work in Section 6.

\section{Observations and data reduction}

\subsection{Dark Energy Survey SN observations}

The DES SN Program searches for SNe by observing 10 different fields in the sky. Observations based on the first year of operations recorded mean cadences ranging between 4.5 and 8 days among all the 10 fields [14]. SN candidates are identified in the difference images, created by subtracting a "reference image" from each observed image. The identified SN candidates undergo further tests and scrutiny to verify that they are genuine SN candidates. The DES SN search pipeline assigns a probability to each identified SN candidate to be of type SN Ia, SN Ibc, SN IIP and so forth.

\subsection{SALT observations and data reduction}

Spectroscopic observations of the $10 \mathrm{SNe}$ Ia presented in this work were conducted with the Robert Stobie Spectrograph (RSS, [15]) on SALT between October 2013 and October 2015. The exposure times for single night exposures for all targets range from 3000 to 4000 seconds including overheads. A few of the targets were visited more than once and their spectra co-added to boost the signal to noise ratio. The RSS was configured with the pg0300 grating to provide a spectral range and resolution of $3800-8200 \AA$ and $R=376$, respectively. Data reduction was performed using the PySALT software [16].

\section{Classification of observed SN candidates}

We typed the observed SN candidates using the supernova identification software SNID [17]. While a SALT SN spectrum similar to that shown in the top pane of Fig. 1 is easily classifiable by SNID, there are others that suffer significant host galaxy light contamination such as that shown in the bottom pane, which SNID is unable to classify successfully. This eventuality prompted us to look into ways of extracting the true SN spectrum from a contaminated observation. While another existing classification software called superfit [18] provides some way of dealing with host galaxy blended SN spectra, it runs on $\mathrm{IDL}^{1}$ which is not open-source. This hurdle got us to look into developing an open-source platform classification software, similar in functionality to superfit.

We have developed a Bayesian inference template-based fitting SN classification method that utilises the Markov Chain Monte Carlo (MCMC) Metropolis-Hastings algorithm [19, 20] and runs in python. The details of the method are to be presented in a separate paper. However, we show in Fig. 2 its result of classifying the same spectrum shown in the top pane of Fig. 1, using the Peter Nugent SN spectral templates ${ }^{2}$ and the Kinney-Calzetti spectral atlas of galaxy templates ${ }^{3}$.

Notice however that the two input spectra in the top panes of Figs. 1 and 2 do not look identical, even though for the same object. The reason is the input and template spectra in the SNID software are wavelength re-binned onto a common wavelength logarithmic scale and continuum subtracted. A bandpass filter is then applied to both to remove low-frequency residuals that result from the

\footnotetext{
${ }^{1}$ IDL stands for Interactive Data Language, a programming language developed by Exelis Visual Information Solutions http://www.exelisvis.com/

${ }^{2}$ https://c3.lbl.gov/nugent/nugent_templates.html

${ }^{3}$ http://www.stsci.edu/hst/observatory/crds/cdbs_kc96.html
} 
continuum subtraction and also to remove high-frequency noise components [17]. Except for rebinning the template spectra to match the wavelength scale of the input spectrum, our method does not perform the rest of the SNID operations to the input and template spectra as that results in the loss of some of the spectral details we care about such as the encoded host galaxy extinction information.
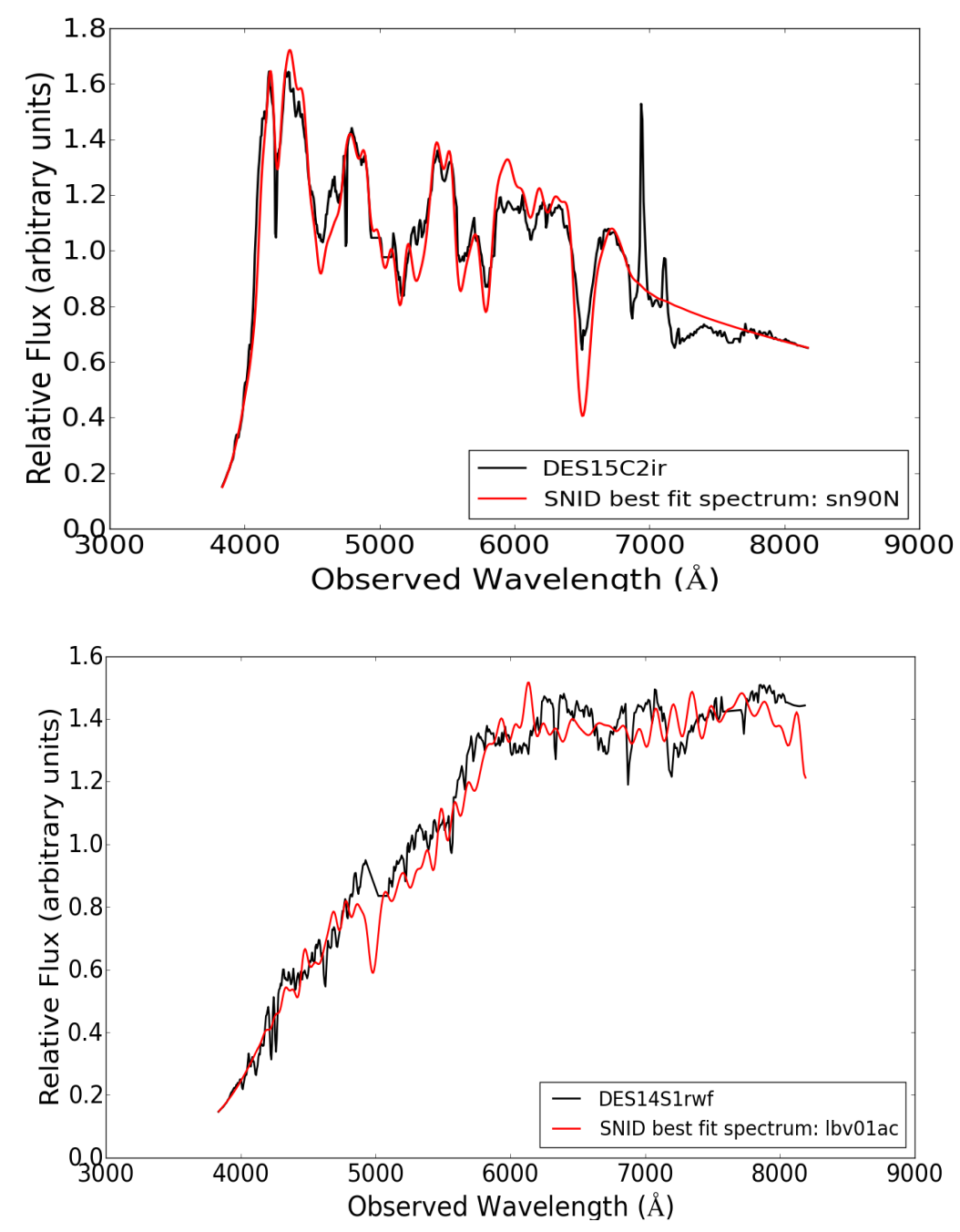

Figure 1: Top: Spectrum of a DES SN candidate "DES15C2ir" taken with SALT and classified with SNID as SN Ia. The SNID best fit spectrum (red) reveal this to be a SN Ia at $z=0.057$ with epoch $t=+3$ ( 3 days after $B$-band peak brightness); Bottom: Spectrum of a DES SN candidate "DES14S1rwf" heavily contaminated with light from its host galaxy. Even though a fraction of the SN flux is contained in the spectrum, SNID incorrectly types it to be that of a luminous blue variable star.

\section{EW measurements of SN Ia spectral features and preliminary results}

To measure the EW of a SN Ia spectral feature, a straight line is initially fitted between two local maxima that bound a given feature. Table 1 of [21] defines the wavelength limits within which the local maxima have to be located for any given feature. The EW is then given by the area 

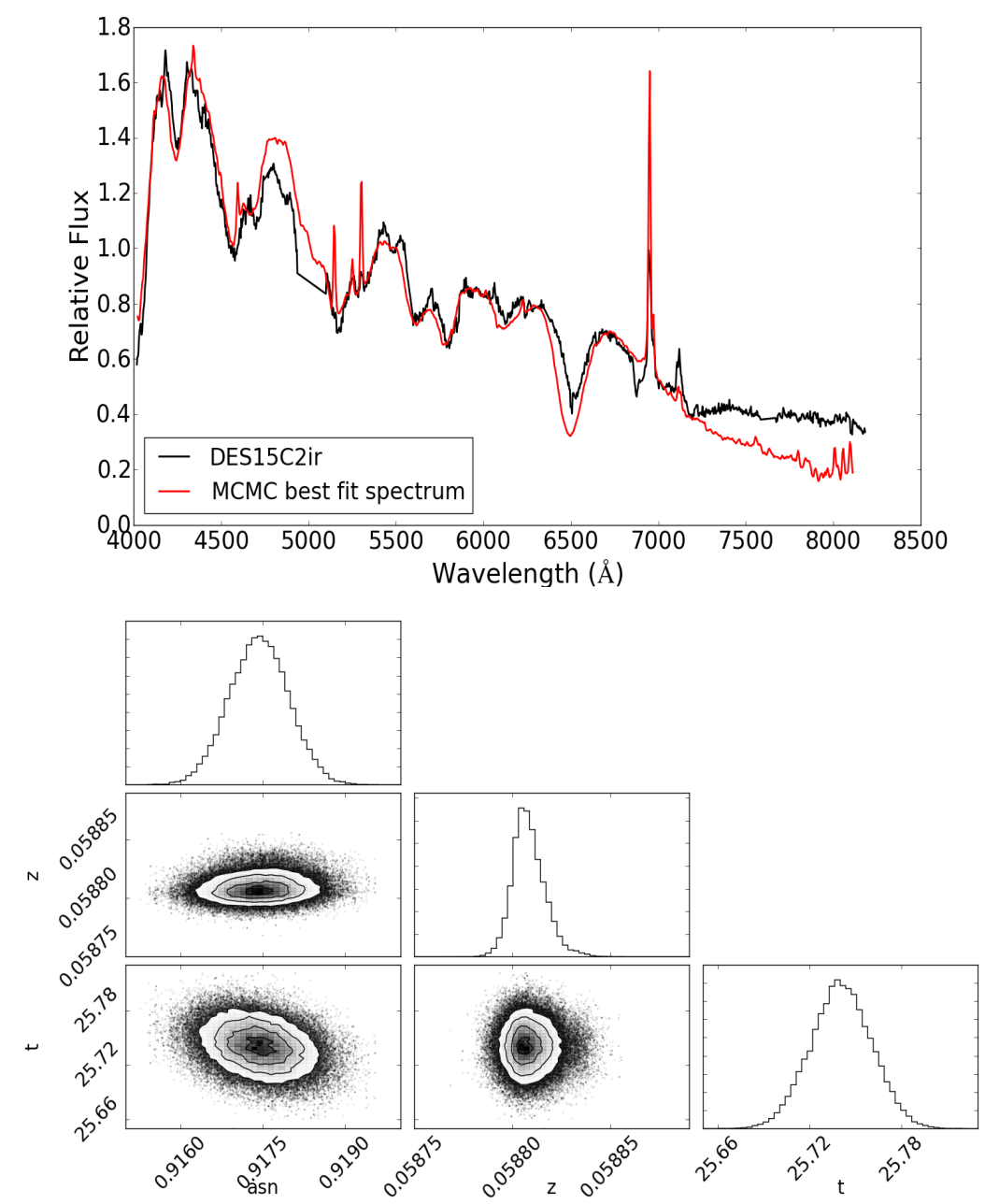

Figure 2: Top: SALT spectrum of DES15C2ir classified with our MCMC algorithm as SN Ia. The best fit galaxy template has less flux towards the red end of spectrum-from $\sim 7250 \AA$ Åonwards—resulting in a poor fit to that portion of the spectrum; Bottom: Histograms and $2 \mathrm{~d}$ contour plots of the 3 parameters we fit for: fraction of SN flux in the spectrum denoted as asn and sometimes as $\alpha$, SN epoch $t$ in days relative to the time of SN explosion and SN redshift $z$. Our MCMC classification recovered $z=0.059, \alpha=0.918$ and $t \sim 25.7$, which translates into $\sim 5.7$ days after $B$-band peak brightness. These are consistent with the SNID recovered parameters in the top pane of Fig 1.

under the fitted line called "pseudo-continuum" [21] and obtained from computing the sum in Eq. (4.1):

$$
E W=\sum_{i=1}^{N}\left(1-\frac{f_{\lambda}\left(\lambda_{i}\right)}{f_{c}\left(\lambda_{i}\right)}\right) \Delta \lambda_{i}
$$

where $\lambda_{i}$ are the centres of the wavelength bins of sizes $\Delta \lambda_{i}$ and $f_{c}\left(\lambda_{i}\right)$ and $f_{\lambda}\left(\lambda_{i}\right)$ are fluxes determined from the pseudo-continuum at $\lambda_{i}$ and flux in bin $i$, respectively. Eq. (4.2) gives the $1 \sigma$ statistical uncertainties in the measured $E W$ values:

$$
\sigma_{E W}^{2}=\sum_{i=1}^{N}\left(\left\{\frac{\sigma_{f}\left(\lambda_{i}\right)}{f_{c}\left(\lambda_{i}\right)}\right\}^{2}+\left\{\frac{f_{\lambda}\left(\lambda_{i}\right)}{f_{c}\left(\lambda_{i}\right)^{2}}\right\}^{2} \cdot \sigma_{c i}^{2}\right) \Delta \lambda_{i}^{2},
$$


where $\sigma_{c i}$ and $\sigma_{f}$ are the uncertainties in the measured fluxes $f_{c}$ and $f_{\lambda}$, respectively. $\sigma_{c i}$ is derived via error propagation of the estimated errors in the slope and intercept of the selected pseudocontinuum. This is done at the points that we measure the values of $f_{c}$. Table 1 shows the results of our measured EWs and the associated errors for the $\mathrm{Ca}$ II H\&K feature ( $\mathrm{EW}_{\mathrm{Ca}}$ for short) and $\mathrm{Si}$ II $4000 \AA$ feature (EW $\mathrm{Ei}_{\mathrm{Si}}$ for short) using Eqs (4.1) and (4.2), for the $10 \mathrm{SNe}$ Ia in our sample.

Table 1: EW measurements of 10 SALT spectra of DES SN Ia candidates taken between October 2013 and October 2015

\begin{tabular}{ccccc}
\hline \hline SN & $z$ & Epoch $t^{a}$ (days) & $\mathrm{EW}_{\mathrm{Ca}}(\AA)$ & $\mathrm{EW}_{\mathrm{Si}}(\AA)$ \\
\hline DES13X1cpt & 0.149 & 0 & $104.7 \pm 6.1$ & $7.4 \pm 2.7$ \\
DES13X1kae & 0.149 & 0 & $101.5 \pm 3.8$ & $10.2 \pm 4.2$ \\
DES13C1juw & 0.2 & 0 & $50.7 \pm 3.1$ & $19.5 \pm 8.5$ \\
DES13C2acmj & 0.114 & +35 & $97.3 \pm 2.5$ & $71.5 \pm 4.3$ \\
DES14X1oes & 0.30 & +7 & $129.2 \pm 4.5$ & $27.7 \pm 4.7$ \\
DES14E1rph & 0.105 & +8 & $146.8 \pm 6.1$ & $31.5 \pm 7.9$ \\
DES15C2ir & 0.058 & +5 & $114.7 \pm 8.4$ & $16.9 \pm 2.5$ \\
DES15X3kqv & 0.1421 & 0 & $121.1 \pm 2.8$ & $14.5 \pm 3.1$ \\
DES15S2kwq & 0.237 & +5 & $97.9 \pm 2.9$ & $b$ \\
DES15E1kwg & 0.1049 & 0 & $119.0 \pm 3.1$ & $11.5 \pm 1.8$ \\
\hline
\end{tabular}

$a$ - Relative to $B$-band peak brightness

$b$ - We do not have a reliable $\mathrm{EW}_{\mathrm{Si}}$ measurement as the feature falls into the first SALT CCD gap at this redshift.

In the top pane of Fig. 3 is a SALT spectrum of "DES15E1kwg", one of the $10 \mathrm{SNe}$ Ia in our sample. Shown in the spectrum as green and magenta filled-in regions are the Ca II H\&K and Si II $4000 \AA$ absorption features each bounded at the top by its fitted pseudo-continuum. The area covered by the filled-in region is the EW value of the feature. We show in the bottom pane a comparison of our measurements to those of [11], performed on a low-redshift sample of $76 \mathrm{SNe}$ Ia spectra from the Nearby Supernova Factory, taken within 2.5 days of $B$-band peak brightness. To enable a direct comparison of the results, we only plot 5 of our 10 data points as half our sample size consists of SNe Ia with epochs $>2.5$ days after $B$-band peak brightness, as Table 1 shows.

\section{Discussion}

Four of our $5 \mathrm{EW}$ measurements within 2.5 days of $B$-band peak brightness appear to be consistent with the [11] results, with the 5th measurement appearing to be somewhat an outlier. While results are preliminary, the 4 measurements appear to suggest that the spectroscopic lightcurve standardization techniques applied to low-redshift SN Ia samples involving the use of $E W$ measurements would yield similar results when applied to intermediate-redshift SNe Ia. This would put tight constraints on SN Ia systematics which could lead to significant improvements in the accuracy of estimating cosmological parameters from SNe Ia data.

Application of our MCMC classification method to real data has shown to return satisfactory results thus far which is very exciting and encouraging as the realisation of its full potential translates into successful classification of more SALT SN spectra that we currently are unable to classify with SNID due to significant host galaxy contamination. 

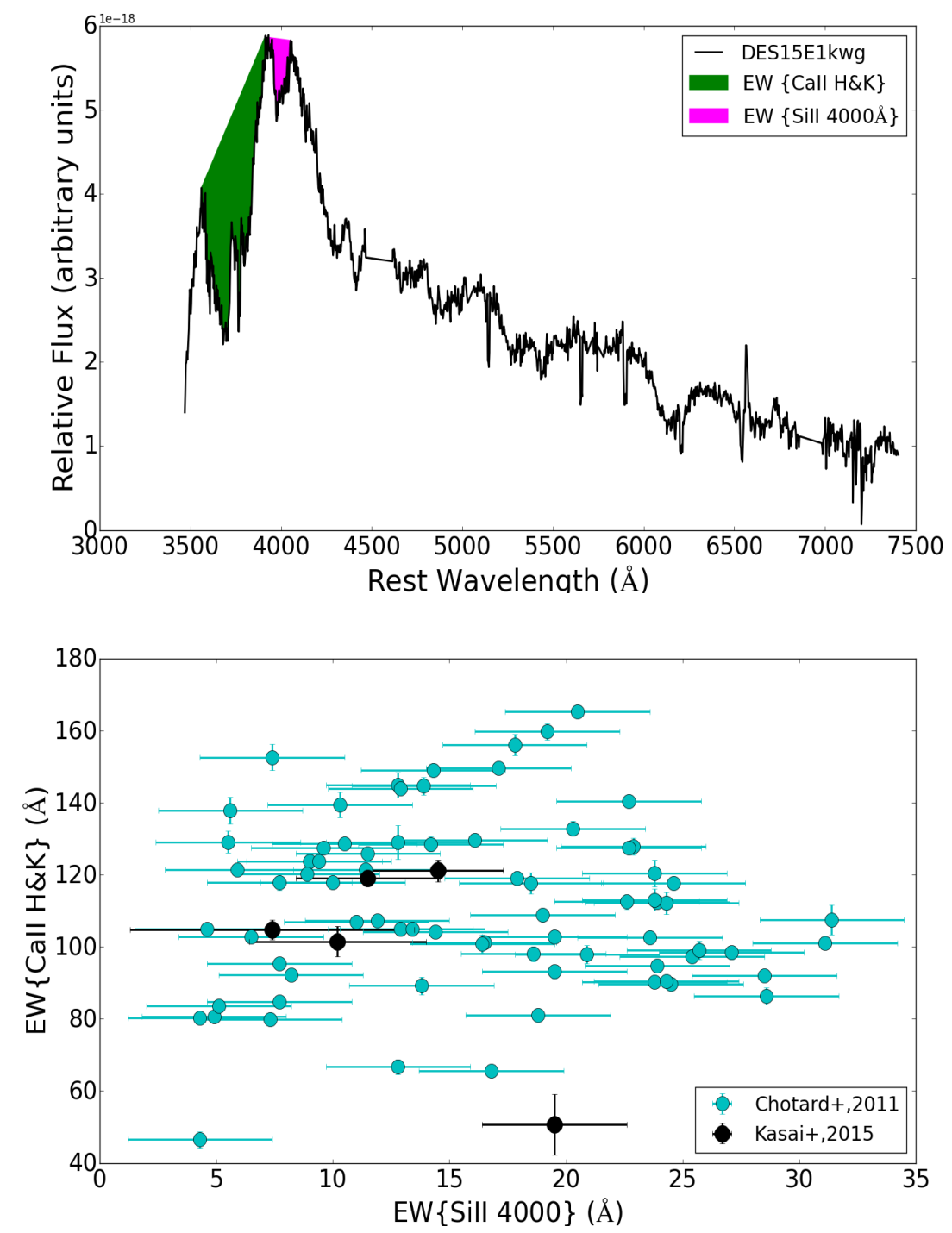

Figure 3: Top: A SALT spectrum of "DES15E1kwg" highlighting in green and magenta filled-in regions the Ca II H\&K and Si II 4000A absorption features used in EW measurements; Bottom: Correlation of the $\mathrm{EW}_{\mathrm{Ca}}$ and $\mathrm{EW}_{\mathrm{Si}}$ of the [11] measurements (cyan) versus our measurements (black) for $5 \mathrm{SNe}$ Ia in the redshift range $0.1<z<0.3$, all with epochs around $B$-band peak brightness $(t \sim 20$ days).

\section{Conclusion and future work}

We continue to conduct spectroscopic follow-up of DES SN candidates with SALT building towards our targeted publishable sample of 25-30 SNe Ia in the redshift range $0.1<z<0.3$. The successful completion of our MCMC classification technique will help increase our sample size, enabling us to test further at intermediate redshifts the spectroscopic SN Ia standardization techniques (including EWs, flux ratios and SN ejection velocities) developed using low-redshift samples as well as to investigate the nature of dust extinction in the host galaxies of intermediateredshift SNe Ia. This will reveal whether or not there is any evidence of evolution in the spectral behaviour of SNe Ia with redshift and could lead to the identification of more exciting spectral and light-curve correlations with a great potential to improve our current understanding of the nature of 
dark energy and other cosmological parameters.

\section{References}

[1] A. G. Riess et al., Observational Evidence from Supernovae for an Accelerating Universe and a Cosmological Constant, AJ 116 (1998) 1009 [arXiv: astro-ph/9805201]

[2] S. Perlmutter et al., Measurements of $\Omega$ and $\Lambda$ from 42 High-Redshift Supernovae, ApJ 517 (1999) 565 [arXiv:astro-ph/9812133]

[3] M. Betoule et al., mproved cosmological constraints from a joint analysis of the SDSS-II and SNLS supernova samples, A\&A $\mathbf{5 6 8}$ (2014) A22 [arXiv: 1401.4064 ]

[4] M. M. Phillips, The absolute magnitudes of Type IA supernovae, ApJL 413 (1997) 105

[5] A. V. Filippenko, Optical Spectra of Supernovae, ARA\&A 35 (1997) 309

[6] J. Guy et al., SALT2: using distant supernovae to improve the use of type Ia supernovae as distance indicators, A\&A 466 (2007) 11 [arXiv: astro-ph/0 701828 ]

[7] S. Jha et al., Improved Distances to Type Ia Supernovae with Multicolor Light-Curve Shapes: MLCS2k2, ApJ 659 (2007) 122 [arXiv: astro-ph/ 0612666 ]

[8] J. Cardelli et al., The relationship between infrared, optical, and ultraviolet extinction, ApJ 345 (1989) 245

[9] R. Tripp, A two-parameter luminosity correction for Type IA supernovae, A\&A 331 (2011) 815

[10] R. Kessler et al., First-Year Sloan Digital Sky Survey-II Supernova Results: Hubble Diagram and Cosmological Parameters, A\&A 185 (2009) 32 [arXiv: 0908 . 4274]

[11] N. Chotard et al., The reddening law of type Ia supernovae: separating intrinsic variability from dust using equivalent widths, A\&A 529 (2011) L4 [arXiv: 1103.5300]

[12] S. Bailey et al., Using spectral flux ratios to standardize SN Ia luminosities, A\&A 500 (2009) L17 [arXiv:0905.0340]

[13] S. Blondin et al., Do spectra improve distance measurements of Type Ia supernovae?, A\&A 526 (2011) A81 [arXiv:1012.0005]

[14] H. T. Diehl et al., The dark energy survey and operations: year 1, SPIE Astronomical Telescopes+ Instrumentation, International Society for Optics and Photonics, (2014) 91490V

[15] E. B. Burgh et al., Prime Focus Imaging Spectrograph for the Southern African Large Telescope: optical design, SPIE 4841 (2003) 1463

[16] S. M. Crawford et al., PySALT: the SALT science pipeline, SPIE 7737 (2010) 25

[17] S. Blondin \& L. J. Tonry Determining the Type, Redshift, and Age of a Supernova Spectrum, ApJ 666 (2007) 1024 [arXiv:0709.4488]

[18] A. D. Howell et al., Gemini Spectroscopy of Supernovae from the Supernova Legacy Survey: Improving High-Redshift Supernova Selection and Classification, ApJ 634 (2005) 1190 [arXiv:astro-ph/0509195]

[19] N. Metropolis et al., Equation of State Calculations by Fast Computing Machines, J. Chem. Phys. 21 (1953) 1087

[20] W. K. Hastings, Monte Carlo sampling methods using Markov chains and their applications, Biometrika 57(1) (1970) 97

[21] G. Garavini et al., Quantitative comparison between type Ia supernova spectra at low and high redshifts: a case study, A\&A 470 (2007) 411 [arXiv : astro-ph/0703629] 